\title{
Allochrony as a potential driver for reproductive isolation in adaptive radiations of European whitefish ecomorphs
}

Julie Bitz-Thorsen, Katja Häkli, Shripathi Bhat, Kim Præbel

Norwegian College of Fishery Science, UiT - The Arctic University of Norway, N-9037 Tromsø, Norway

Running head title: Allochrony as post-zygotic extrinsic isolation mechanism in Coregonus

Corresponding author:

Julie Bitz-Thorsen, Norwegian College of Fishery Science, UiT - The Arctic University of Norway.

Email: julie.bitz-thorsen@uit.no

Word count abstract: 249

Word count main text: 4273

Number of references: 55

Number of tables: 2 (+ 1 supplementary)

Number of figures: 2 


\section{Abstract}

In northern Fennoscandian lakes, monophylogenetic lineages of postglacial fishes are radiating into several adaptive forms, but the speciation process is still at an incipient stage. The speciation process has received increased attention over the years, but the underlying mechanisms and drivers are still debated and poorly understood. European whitefish (Coregonus lavaretus [L.]) is the most abundant fish species in these lakes and has evolved into several ecomorphs adapted to different trophic niches and habitats. Genetic divergence has been observed among these ecomorphs, but the mechanism(s) responsible for the ongoing build-up of reproductive isolation has still to be revealed. As these systems are young in evolutionary time $(<10 \mathrm{kyr})$, pre- and post-zygotic extrinsic isolation mechanisms are thought to be more likely to contribute to the reproductive isolation than intrinsic isolation mechanisms. We determined the gonadosomatic index (GSI) of three ecomorphs in two replicated lake systems and used GSI as a proxy to investigate the pre-zygotic isolation mechanism, allochrony, as a driving factor of divergence in this adaptive radiation of whitefish. We found that the three ecomorphs differed in GSI-values within and between lakes, suggesting different spawning times of the ecomorphs. We also show that males of one ecomorph had equal onset of maturity as another ecomorph, giving novel insights into the ongoing gene flow observed between ecomorphs. The result supports allochrony as a driver for the divergence process of whitefish ecomorphs, but more evidence is still needed to rule out that the three ecomorphs make use of different spawning grounds.

Keywords:

Coregonus lavaretus, whitefish, gonadosomatic index, habitat preference, spawning time, allochrony, ecological speciation 


\section{Introduction}

Adaptive radiation is the rapid evolutionary divergence of individuals from a common ancestor into a variety of adaptive forms (Futuyma 1998) that exploit different ecological niches (Grant \& Grant 2008). Understanding what processes drive and maintain an adaptive radiation is a central question in evolutionary ecology. The availability of various ecological opportunities in an ecosystem allows for different niches that may favour different behavioural and morphological adaptations. The association between a particular morphology and a specific niche is recognized as an important factor in adaptive radiation (Schluter 2000), and in fish, this association is often related to foraging traits, e.g. head shape and trophic niche (Schluter 1996). These associations can lead to genetic divergence and reproductive isolation of different adaptive forms (Rundle \& Nosil 2005, Schluter 2000). Pre-zygotic and post-zygotic isolation are different isolation mechanisms that can result in reproductive isolation. Pre-zygotic isolation involves spatial and temporal isolation (e.g., different spawning sites and time) and sexual selection (Ritchie 2007, Taylor \& Friesen 2017), while postzygotic isolation includes ecological inviability (extrinsic), hybrid inviability, and sterility (intrinsic) (Coyne \& Orr 2004). Despite extensive efforts in understanding why and how reproductive isolation accumulates in adaptive radiations, the exact drivers still remain to be identified for many species.

Polymorphic populations are commonly found in several freshwater fish species in postglacial lakes of the northern hemisphere. The fish species inhabiting these lakes have shown rapid $(<10$ kyr), convergent phenotypic divergence and adaptive radiation into multiple ecomorphs (Häkli et al. 2018, Østbye et al. 2005a, Østbye et al. 2006, Schluter 2000, Taylor 1999). European whitefish (Coregonus lavaretus [L.], referred to as whitefish further on) is a widely distributed fish species in Europe and is highly abundant in northern Fennoscandia. Here it has diverged from a monophyletic 
lineage (Østbye et al. 2005a) into distinct ecological morphs through adaptive radiation (Svärdson 1979, Østbye et al. 2006). Three distinct ecomorphs of whitefish have been described (Kahilainen \& Østbye 2006, Siwertsson et al. 2010), which show clear differences in niche utilization and trophic morphology, e.g. head shape and gill rakers (Amundsen et al. 2004, Harrod et al. 2010, Kahilainen \& Østbye 2006), and have also been found to differentiate genetically (Præbel et al. 2013a, Siwertsson et al. 2013). However, the reproductive isolation among the ecomorphs is not complete, evidenced by the frequent occurrence of hybrids between the ecomorphs (Bhat et al. 2014).

The three whitefish ecomorphs are associated to the feeding niches found in three main habitats of the lakes; the littoral, pelagic and profundal zones (Kahilainen et al. 2003, Østbye et al. 2006). The ecomorphs are named according to morphology of gill rakers (Kahilainen \& Østbye 2006). The densely rakered whitefish ecomorph (hereafter DR whitefish) resides mainly in the pelagic habitat and is a zooplanktivorous specialist, the large sparsely rakered whitefish (LSR whitefish) mainly feeds on benthic macroinvertebrates in the littoral habitat, and the small sparsely rakered whitefish (SSR whitefish) is mainly found in the profundal habitat feeding on benthic invertebrates (Harrod et al. 2010). In a recent study it was found that initial divergence of the ecomorphs into different habitats was a result of the presence of pike (Esox lucius) in the lakes (Öhlund et al. unpublished). The presence of pike very likely has caused the whitefish to either refuge into new, but less suitable habitats, or maximize growth to reach a safe size. Apart from variation in food resources and basal sources of energy in the three principal habitats (Harrod et al. 2010), they also differ with regard to their thermal conditions (Hayden et al. 2013; Evans et al. 2014). Thermal stratification of the water column in postglacial lakes means that littoral and pelagic habitats undergo large seasonal changes in water temperature from $10-20{ }^{\circ} \mathrm{C}$ in the warm summer 
months and to $0-2{ }^{\circ} \mathrm{C}$ in the cold winter months when covered with ice (Hayden et al. 2013), whereas the profundal habitat display a cold, but stable, temperature of $2-8{ }^{\circ} \mathrm{C}$, throughout the year.

The isolation mechanism driving the ongoing divergence in adaptive radiation of whitefish still has to be identified in these northern systems. This is important in understanding the biological, ecological, and genetic mechanisms involved in their adaptive radiation. Studies on whitefish in more southern Swedish lakes (Öhlund et al. unpublished, Svärdson 1979) suggested spatial and temporal differences in spawning time, but little is known about the exact spawning times and places for the different ecomorphs in northern Fennoscandian lakes. Based on field observations in Lake Paadar of spawning shoals in shallow water, there were indications of the DR whitefish being the first ecomorph to spawn, followed by the LSR whitefish and finally SSR whitefish (K. Kahilainen pers. obs. in Kahilainen et al. 2014). A fourth ecomorph, LDR, is also present in lake Padaar but it occurs in low abundance and is only present in the Pasvik watercourse, not the Alta watercourse where this study was performed. In coregonids, such interspecific variation in spawning time are important factors driving reproductive isolation (Bernatchez et al. 2010, Hudson et al. 2007, Svärdson 1979). The late spawning time of SSR whitefish may reflect the dark and stable cold temperatures in profundal habitats which slows down metabolic processes (Ohlberger et al. 2008). Physiological adaptation, such as metabolic and maturation processes, to a particular light and thermal regime should differ substantially among the ecomorphs, but so far only niche driven and respiration adaptations have been suggested as drivers of phenotypic divergence and reproductive isolation in northern post-glacial lakes (Evans et al. 2014, Harrod et al. 2010, Keller \& Seehausen 2012, Østbye et al. 2006). 
In this study, we investigated the pre-zygotic isolation mechanism(s) among the three whitefish ecomorphs. The challenge of revealing reproduction in these northern systems, compared to more southern lakes e.g. in Sweden, Denmark, and the European Alps, is that the spawning takes place in late October to December, when the lake ice is too thin to work on, but too thick for boating. Based on our own field observations, information from locals, and the presence of hybrids between the ecomorphs (Bhat et al. 2014), it seems that all three ecomorphs mainly share the same spawning ground(s) in the littoral zone, and with the knowledge of whitefish ecomorphs' thermal niches, we hypothesized that differences in spawning time (allochrony) acts as the main driver of reproductive isolation. This differs from the spatial divergence in spawning grounds observed in other more southern whitefish systems (Østbye et al. 2005b; Vonlanthen et al. 2009). We sampled European whitefish ecomorphs in two replicated northern lakes, as close to their natural spawning time as possible, and calculated a gonadosomatic index (GSI) as a measurement of sexual maturity. GSI has previously been used to determine sexual maturity, seasonal changes, and reproduction timing in fish (e.g. Flores et al. 2015, McQuinn 1989, Valdés et al. 2004). Seasonal changes in GSI of female whitefish (of a morph equivalent to the DR morph in this study) in lake Constance, Germany, showed that the GSI increased steadily over the summer and autumn until it reached a maximum, just before spawning in the winter (Rösch 2000). To our knowledge, no previous study has used GSI to investigate allochrony in a polymorphic species, and by comparing sexual maturation of the three whitefish ecomorphs, our goal was to infer whether the whitefish ecomorphs display differences in spawning time.

\title{
Materials and Methods
}

\author{
Sample collection
}


In October 2016, over a period of seven days, two lakes located in the northern Fennoscandia were sampled for European whitefish ecomorphs, Lake Stuorajávri $\left(69^{\circ} 06^{\prime} \mathrm{N}, 22^{\circ} 49^{\prime} \mathrm{E}\right)$ and Lake Suohpatjávri $\left(68^{\circ} 56^{\prime} \mathrm{N}, 23^{\circ} 05^{\prime} \mathrm{E}\right)$. Stuorajávri covers an area of $24 \mathrm{~km} 2$ with a maximum depth of $30 \mathrm{~m}$, and Suohpatjávri covers $2 \mathrm{~km} 2$ with a maximum depth of $25 \mathrm{~m}$. The two lakes are oligotrophic, harbour six fish species in addition to the whitefish, and have relatively equal distribution of shallow and deep areas. Both lakes have three principal habitats: the littoral habitat (shore water, close proximity to the bottom, $<10 \mathrm{~m}$ depth; $>1 \%$ of light at surface), the profundal habitat (close proximity to the bottom, $>10 \mathrm{~m}$ depth; <1\% of light at surface), and the pelagic habitat (open water, 0-6 m depths). Three different ecomorphs of whitefish are found in both of these lakes: DR, LSR and SSR whitefish (Siwertsson et al. 2013) (Figure 1). Fish sampling was performed with standardized gillnets of different mesh sizes, 10, 12.5, 15, 18.5, 22, 26, 35, and 45 $\mathrm{mm}$. Benthic gillnets $(1.5 \mathrm{~m}$ high) were used in the littoral and profundal zones, whereas the pelagic habitat was sampled using 6-m-high floating nets. A total of 364 whitefish were collected (Table 1). For each individual fish, total body weight and gonad weight were determined in the field laboratory using a Sartorius BP 310s scale and subsequently used to calculate the gonadosomatic index $(\mathrm{GSI}=\text { [testis or ovary weight / body weight }]^{* 100)}$ per individual (Table 1$)$. We further recorded the sex (female/male), sexual maturity (immature/mature), and total length for all individuals. Figure 1 shows a subsample of gonads for each whitefish ecomorph documented using a Nikon D610 camera body mounted with a Nikon 16.0-35.0 mm f/4.0ED lens. A fin tissue sample was also collected and stored in $96 \%$ ethanol at $-20 \circ \mathrm{C}$ for later genetic analysis.

[Insert Figure 1 near here]

[Insert Table 1 near here] 


\section{Ecomorph assignment and discrimination}

A phenotypic analysis of the fish was performed in the field, where each individual was classified as DR, LSR or SSR whitefish according to appearance, head and body shape, and a visual evaluation of the gill raker morphology (Amundsen et al. 2004, Kahilainen \& Østbye 2006). Ecomorph assignment was further verified in the laboratory by assessing the first left branchial arch under a microscope and counting the number of gill rakers (Table 1). We did not distinguish between male and female when counting of gill rakers.

To assess genetic divergence between the ecomorphs we followed the protocol of Præbel et al. (2013b) using a panel of 22 microsatellite markers. The data was screened for scoring errors, allele dropouts and null alleles using Micro-Checker 2.2.3 (van Oosterhout et al. 2004). Deviations from Hardy-Weinberg equilibrium (HWE) and linkage disequilibrium were tested using exact tests (Guo \& Thompson 1992) as implemented in GenePop 4.0 (Rousset 2007). Sequential Bonferroni corrections (BFC) was used to correct pairwise comparisons for multiple comparisons following Rice (1989). Genetic divergence among morphs was estimated using the pairwise.fst function in the Adegenet R-package (Jombart 2008), which computes Nei’s estimator of pairwise Fst, and tested for significance using 1000 permutations. For the genetic divergence analysis, we used both immature and mature individuals.

\section{Preliminary analyses and quality control of GSI-maturity data}

For each lake, fish were divided into groups based on ecomorph, sex and sexual maturity (immature and mature). Division of sex was necessary because the weight of the gonads differs greatly between males and females, and the assessment of sexual maturity was necessary because we only were interested in using mature individuals for this study. Individuals that did not fulfil these criteria were excluded from the analyses, leaving 168 females and 148 males for the statistical 
analyses. Gonadosomatic index was calculated and used as a measurement for sexual maturity, i.e. the advancement of sexual maturity towards spawning, because all individuals were mature. This GSI-maturity functioned as our proxy for differences in spawning time between whitefish ecomorphs. This means that the GSI-values are under the assumption that greater GSI-values equals a more sexually mature fish, and consequently, has an earlier spawning time, than a fish with a lower GSI-value. The GSI-values were transformed using the logarithmic function to more closely meet the assumption of linear models and because our dependent variable is a proportion (Baum 2008). An overview of the number of mature individuals, percentage of maturity and the average GSI for each ecomorph in the two lakes is found in Table 1.

\section{Determination of variation in GSI-maturity among whitefish ecomorphs}

For the statistical data analyses of GSI-maturity among ecomorphs we carried out a two-way analysis of variance (ANOVA) using R v.1.0.44 (R Core Team 2016). The first step was model selection using the MuMin package in R (Barton 2017) to find the linear model that best explained the differences in maturity of whitefish $(\log 10(\mathrm{GSI}))$. Using a factorial design of relevant variables, we tested 16 different linear models. The additive model of two factors (or independent variables), population (ecomorphs) and lake, was the most parsimonious fit according to Akaike's Information Criteria (AIC) statistics for model selection. This model had, for both sexes, the lowest AIC-value with the lowest degrees of freedom and most weight to it. Next step was a control of the model to check if the data fitted a normal distribution by plotting a Q-Q plot. To detect the presence of outliers in our dataset we used the Interquartile Range Rule (IQR). For the males only, the data contained nine outliers outside 1,5 x IQR that skewed the distribution and thus needed to be removed from the dataset. This left us with 139 males for further analysis. The nine outliers removed from the male dataset consisted of 8 individuals with much lower GSI-values, indicating 
they were wrongly assigned as sexually mature in the field when in fact they were immature. One individual had a much higher GSI-value than average, which may be the result of a typo-error during the field work. The gonad weight of this particular individual is $>50 \%$ more than average. After the preliminary steps, the ANOVA statistics were performed and finally, post hoc analyses using pairwise comparisons of least-squares means for the specified factors in the linear model using the estimability and lsmeans packages in R (Lenth 2016a, 2016b).

Additionally, we caught an unusually low number of SSR whitefish in Lake Suohpatjávri, only one mature male in total. This was clearly not enough to do proper statistical analysis, but we included the individual anyway because this individual was in fact not an outlier in the model control step allowing us to obtain some insight into the GSI-values of male SSR whitefish.

[Insert Table 2 near here]

\section{Results}

\section{Genetic differentiation among whitefish morphs}

The number of alleles at each microsatellite locus ranged from 2 to 21 across populations (Table S1). Micro-Checker detected homozygote excess due to possible null alleles in four of 22 tested loci: Cocl_lav27 (Stuorajávri, DR whitefish), and BWF1, ClaTet06 and Cocl_lav10 (Stuorajávri, SSR whitefish) (Table S1). Although none of the possible null alleles were found in more than one population at a time, those four loci were excluded from further analysis. Deviations from HWE were indicated in 1 out of 108 tests $(0.9 \%)$ after sequential Bonferroni corrections, which is less than expected by chance (5\%). Out of 918 tests, significant linkage disequilibrium was found in three $(0.3 \%)$ loci comparisons after Bonferroni correction. Pairwise FsT values between populations 
were small yet statistically significant, ranging from 0.011 to 0.059 , where the smallest genetic difference was found between LSR whitefish in different lakes (Table 2).

\section{Field-determined maturity of whitefish morphs}

The abundance of mature individuals differed both between the ecomorphs and lakes (Table 1). When individuals of both sexes were combined, we found a pattern that most often the DR whitefish had more mature individuals than the LSR whitefish, which again had more mature individuals than the SSR whitefish. The only exception being females in Stuorajávri, where 85.2\% of DR whitefish were mature versus the $94.7 \%$ of LSR whitefish (Table 1). Between lakes, there was a higher proportion of mature whitefish caught in Stuorajávri than in Suohpatjávri, especially for the LSR and SSR whitefish. The DR whitefish appeared to have an equal proportion of mature individuals between the lakes.

\section{Variation in GSI-maturity of whitefish ecomorphs}

The two-way ANOVA showed that there are significant differences of the advancement of sexual maturity (log10GSI) between the three distinguished whitefish ecomorphs for both sexes (females: $\mathrm{F}_{2,165}=17.359, P<0.001$; males: $\left.\mathrm{F}_{2,135}=9.097, P<0.001\right)$. There were also significant differences in GSI-maturity for both sexes between the two lakes (females: $F_{1,165}=15.293, P<0.001$; males: $\left.\mathrm{F}_{1,135}=46.838, P<0.001\right)$. For the females, the DR whitefish was significantly more mature than the LSR whitefish $\left(P<0.001, R_{2}=0.2186\right)$ and the SSR whitefish $\left(P<0.001, R_{2}=0.2186\right)$, but the LSR whitefish was not significantly more mature than the SSR whitefish $\left(P=0.597, R_{2}=0.2485\right)$. For the males, there was no difference in maturity between DR whitefish and LSR whitefish $(P=0.752$, $\left.R_{2}=0.310\right)$, but both the DR whitefish $\left(P<0.001, R_{2}=0.310\right)$ and LSR whitefish $(P<0.001$, $\left.R_{2}=0.2615\right)$ were significantly more mature than the SSR whitefish. 
For the post hoc analyses, we did pairwise comparisons using least-square means of $\log 10(\mathrm{GSI})$ on the specified factors in the linear model (Figure $2 \mathrm{a}$ and $2 \mathrm{~b}$ ). These analyses contrasted the advancement of sexual maturity of the whitefish ecomorphs individually from each lake. For the female whitefish, the whitefish from Stuorajávri were significantly more mature compared with whitefish in Suohpatjávri. Also, for females, the DR whitefish in both lakes were more mature compared to the LSR whitefish and SSR whitefish, which in turn were equally mature. For the male whitefish, as with the females, the whitefish in Stuorajávri were more mature compared with whitefish in Suohpatjávri. Furthermore, the male DR whitefish and LSR whitefish were equally mature, whereas they were significantly more mature than SSR whitefish in both lakes.

[Insert Figure $2 \mathrm{a}$ and $2 \mathrm{~b}$ near here]

\section{Discussion}

We found statistically significant differences in the advancement of sexual maturity, based on GSIvalues, between the three genetically and morphologically divergent ecomorphs of whitefish. We demonstrated that the pelagic DR whitefish were generally further advanced in sexual maturity than the littoral LSR whitefish, which in turn were more mature than the profundal SSR whitefish at the moment of capture. This pattern was prevalent in both of the sampled post-glacial lakes and for both sexes. The pattern was also evident from the morphological appearance of the gonads of the ecomorphs, where DR whitefish had far more developed oocytes than the other ecomorphs at the moment of capture (Figure 1). However, our results do not permit inference about the temporal pattern of gonad development, nor do they provide information about ecological factors that drive the maturation. Rösch (2000) investigated, over a five-year period, seasonal changes in GSI of the 
Blaufelchen whitefish (morph equivalent to the DR morph in this study) in Lake ConstanceObersee and found that GSI increased from low values in summer to about $24 \%$ of the body weight immediately before spawning in December. A similar result was obtained for Pollan whitefish in Lough Neagh, Ireland (Wilson \& Pitcher 1983), suggesting that GSI is a useful measure of sexual maturity in Coregonus. The whitefish radiations in Northern Fennoscandia have been shown to be driven by ecological opportunity (Siwertsson et al. 2010), where each of the ecomorphs have adopted a life-history and specialization to each of the principal lake niches (littoral, pelagic, profundal). The DR whitefish feed on pelagic prey, which leads to the smallest body size and earliest sexual maturation, the LSR whitefish feed on littoral benthic macroinvertebrates, which leads to large size and late sexual maturation, and the SSR whitefish feed on profundal benthic prey and grow to intermediate body size and late sexual maturation (Harrod et al. 2010; Hayden et al., 2013; Kahilainen et al. 2003, 2005; Østbye et al. 2006). Our results of GSI-estimated maturity extend the understanding of the general life history characteristics of the ecomorphs by adding a likely spawning chronology, similar to what has been observed in the field on a single occasion (K. Kahilainen pers. obs. in Kahilainen et al. 2014).

Estimating the advancement of sexual maturity with GSI was based on the assumption that GSI-values increase with increasing sexual maturity and earlier spawning time. According to Ohlberger's theory (2013), the temporal spawning segregation (allochrony) develops because individuals living in different habitats experience different water temperatures and therefore mature at different rates. For whitefish, a discussed mechanism to drive differences in timing of spawning activities, and thus differences in sexual maturity between diverging ecomorphs, is the exposure to different environmental conditions, e.g. light and temperature regimes (Evans et al. 2014, Kahilainen et al. 2014). Adaptation to divergent thermal niches is also likely to be of crucial 
importance to the relative capacity to assimilate the energy required to reach sexual maturity and the development of gonads (Kahilainen et al. 2014). These habitat specific differences reflect what the three whitefish ecomorphs in northern postglacial lakes experience, where the DR, LSR, and SSR whitefish segregate in the pelagic, littoral and profundal habitats, respectively (Østbye et al. 2006, Præbel et al. 2013a, Siwertsson et al. 2013, Svärdson 1979). The relative contribution of ecological and physical factors on timing of sexual maturity for these whitefish ecomorphs remains to be investigated. But, all together, our hypothesis is supported in that the accumulation of genetic differences observed between the three whitefish ecomorphs (Østbye et al. 2006, Præbel et al. 2013a, Siwertsson et al. 2013), is driven by a pre-zygotic extrinsic isolation mechanism, and is best explained by differences in spawning time, allochrony.

The lakes, as local entries, also appear to have an influence on sexual maturity as we found statistically significant difference of the advancement of sexual maturity between the two lakes. The whitefish from Stuorajávri were significantly more advanced in sexual maturity than the whitefish from Suohpatjávri. Stuorajávri is larger $(24 \mathrm{~km} 2)$ and a little deeper (max depth of $30 \mathrm{~m}$ ) compared to Suohpatjávri $(2.0 \mathrm{~km} 2,25 \mathrm{~m})$ and may therefore retain an overall warmer annual water temperature. Increased environmental temperatures are known to accelerate the physiological rate of animals and may induce earlier maturation of the Stuorajávri whitefish. Locals and scientists have also observed Stuorajávri whitefish to spawn in October-December (Pers. Com. Rune Knudsen) and in Suohpatjávri in November-January (Pers. Com. Eleonor Beck). However, further long-term studies are needed to elucidate the relative contribution of light and temperature to the extrinsic postzygotic isolation mechanisms in the northern Fennoscandian whitefish radiations to confirm these observations. 
Where, when, and how the whitefish spawn in nature is evidently important for understanding the evolution of these radiations. Earlier maturation of male whitefish and their presence on the spawning grounds before and after the arrival and spawning of females, have been observed for Pollan whitefish in Lough Neagh, Ireland (Dabrowski 1981). We also found, in both lakes, that male and female DR and male LSR whitefish displayed a similar state of maturity, while the female LSR and male and female SSR whitefish were less mature. This suggests that in these lakes, the temporal chronology of reproduction is: DR whitefish, LSR whitefish, and SSR whitefish. Notably, we show that the male LSR whitefish is mature at the same time as DR whitefish, suggesting a sexspecific route for the ongoing gene flow observed between the whitefish ecomorphs in their adaptive radiation (Bhat et al. 2014, Häkli et al. 2018, Præbel et al. 2013a, Siwertsson et al. 2013). Hybrids between whitefish ecomorphs frequently occur in all northern Fennoscandian lakes, but genetic studies have only observed hybridisation between DR-LSR whitefish (Bhat et al. 2014) and between LSR-SSR whitefish (Præbel et al. unpublished). A study, using genome-wide coverage of SNPs to infer genomic consequences of speciation reversal in whitefish ecomorphs, showed that hybridisation between female DR and male LSR is the most likely route of gene flow between the whitefish ecomorphs (Bhat 2016). The exact mechanism of this hybridisation is not known and a potential explanation could be sneaking behaviour of LSR males, which has been shown in other salmonids (e.g., Baxter et al. 1997, Garcia-Vazquez et al. 2001, Redenbach \& Taylor 2003). However, this behaviour has thus far only been descried for small males and we therefore find it most likely to be caused by an overlap in spawning. Collectively, the ongoing gene flow between whitefish ecomorphs may be explained by sexually mature male LSR whitefish that spawn with mature DR and we propose that male SSR x Female LSR is a possible sex-specific route for gene flow between the SSR and LSR whitefish ecomorphs (Figure $2 \mathrm{a}$ and $2 \mathrm{~b}$ ). These results also support 
our hypothesis that the ecomorphs share spawning ground(s), but are reproductively isolated in time.

The most pronounced difference in terms of trophic ecology, morphology, and reproductive isolation has been found between the SSR and DR whitefish (Harrod et al. 2010, Kahilainen \& Østbye 2006, Præbel et al. 2013a, Siwertsson et al. 2013). Our results also support these observations in both lakes and for both sexes. There was no overlap of GSI-values between the DR and SSR ecomorphs indicating that extrinsic pre-zygotic isolation mechanisms are in play, quite possibly allochrony, and thus drive the diverging populations. Temporal separation can be important in achieving reproductive isolation in incipient ecological speciation (Schluter 2001), where intrinsic post-zygotic isolation mechanisms, such as genomic incompatibility, has yet to be accumulated between the diverging ecomorphs. A recent study by Johannsson et al. (2017) established that spawning window and population age were positively correlated in all ecomorphs of a lake. Hence, the older the lake is, the more prevalent divergence is for the whitefish ecomorphs. Overall spawning time is therefore increased as an intrinsic isolation mechanism to reduce contact and avoid interbreeding. This supports allochrony as a potential main driver for reproductive isolation between whitefish ecomorphs.

In conclusion, we found statistically significant differences in maturity between whitefish ecomorphs. Overall, the pelagic, zooplanktivorous DR whitefish displayed greater sexual advancement than the littoral, benthic feeding LSR whitefish, which again showed greater maturity than the profundal, benthic feeding SSR whitefish. This was the general pattern for both lakes, despite Stuorajávri showing greater proportion of mature individuals than Suohpatjávri. The morphological divergence between the ecomorphs, their adaptation to different thermal niches, and 
sex-specific routes for gene flow between the ecomorphs, all suggest allochrony as a pre-zygotic isolation mechanism that contributes to the adaptive radiation in European whitefish.

\section{Acknowledgements}

We thank UiT The Arctic University of Norway for supporting KP with funding for a PhD scholarship for KH, the Norwegian College of Fishery Science for funding the study, and JBTs fieldwork. The Freshwater Ecology group at UiT is thanked for collaboration during the fieldwork. We also thank two anonymous reviewers for valuable criticism of earlier versions of the manuscript and Kamilla Bentsen for the linguistic corrections of the manuscript.

\section{Data availability}

The data is available from UiT Open Research Data, DOI: xxxx.

\section{References}

Amundsen, P.-A., Bøhn, T., \& Vaga, G. H. (2004). Gill raker morphology and feeding ecology of two sympatric morphs of European whitefish (Coregonus lavaretus) morphs. Annales Zoologici Fennici, 41:291-300.

Bartoń, K. (2017). MuMIn: Multi-Model Inference [R package version 1.40.0]. https://CRAN.Rproject.org/package=MuMIn

Baum, C. F. (2008). Modeling proportions. The Stata Journal, 8:299-303. 
Baxter, J. S., Taylor, E. B., Devlin, R. H., Hagen, J., \& McPhail, J. D. (1997). Evidence for natural hybridization between Dolly Varden (Salvelinus malma) and bull trout (Salvelinus confluentus) in a northcentral British Columbia watershed. Canadian Journal of Fisheries and Aquatic Sciences, 54(2):421-429.

Bernatchez, L., Renaut, S., Whiteley, A. R., Derome, N., Jeukens, J., Landry, L., Lu, Q., Nolte, A. W., Østbye, K., Rogers, S. M., \& St-Cyr, J. (2010). On the origin of species: insights from the ecological genomics of lake whitefish. Philosophical Transactions of the Royal Society B Biological Sciences, 365:1783-1800. https://doi.org/10.1098/rstb.2009.0274

Bhat S., Amundsen, P.-A., Knudsen, R., Gjelland, K. Ø., Fevolden, S.-E., Bernatchez, L., \& Præbel, K. (2014). Speciation Reversal in European Whitefish (Coregonus lavaretus (L.)) Caused by Competitor Invasion. PLoS ONE, 9(3): e91208. https://doi.org/10.1371/journal.pone.0091208

Bhat, S. (2016). Speciation reversal in sympatric eco-morphs of European whitefish (Coregonus lavaretus L.) - phenotypic and genomic consequences ( $\mathrm{PhD}$ dissertation). Retrieved from http://hdl.handle.net/10037/9670

Coyne, J. A., \& Orr, H. A. (2004). Speciation. Sinauer Associates Inc.: Sunderland, MA.

Dabrowski, K. R. (1981). The spawning and early life history of the pollan (Coregonus pollan Thompson) in Lough Neagh, Northern Ireland. Internationale Revue der gesamten Hydrobiologie und Hydrographie, 66:299-326. https://doi.org/10.1002/iroh.19810660303 
Evans, M. L., Præbel, K., Peruzzi, S., Amundsen, P-A., \& Bernatchez, L. (2014). Phenotypeenvironment association of the oxygen transport system in trimorphic European whitefish (Coregonus lavaretus) populations. Evolution, 68:2197-2210. https://doi.org/10.1111/evo.12442

Florés, A., Wiff, R., \& Díaz, E. (2015). Using the gonadosomatic index to estimate the maturity ogive: application to Chilean hake (Merluccius gayi gayi). ICES Journal of Marine Science, 72(2): 508-514. doi:10.1093/icesjms/fsu155

Futuyma, D. J. (1998). Evolutionary biology (3rd ed.). Sinauer Associates Inc.: Sunderland, MA.

Garcia-Vazquez, E., Moran, P., Martinez, J. L., Perez, J., de Gaudemar, B., \& Beall, E. (2001). Alternative Mating Strategies in Atlantic Salmon and Brown Trout. Journal of Heredity, Vol. 92, 2:146-149. https://doi.org/10.1093/jhered/92.2.146.

Grant, P. R., \& Grant, B.R. (2008). How and Why Species Multiply: The Radiation of Darwin's Finches. Princeton University Press, Princeton, NJ.

Guo, S. W., \& Thompson, E. A. (1992). Performing the exact test of Hardy-Weinberg proportion for multiple alleles. Biometrics, 48(2):361-372.

Harrod, C., Mallela, J., \& Kahilainen K. K. (2010). Phenotype-environment correlations in a putative whitefish adaptive radiation. Journal of Animal Ecology, 79(5):1057-1068. https://doi.org/10.1111/j.1365-2656.2010.01702.x 
Häkli, K., Østbye, K., Kahilainen, K. K., Amundsen, P.-A., \& Præbel, K. (2018). Diversifying selection drives parallel evolution of gill raker number and body size along the speciation continuum of European whitefish. Ecology and Evolution, 8(5):2617-2631. https://doi.org/10.1002/ece3.3876

1. Hayden, B., Harrod, C., \& Kahilainen, K. K. (2013). The effects of winter ice cover on the trophic ecology of whitefish (Coregonus lavaretus L.) in subarctic lakes. Ecology of Freshwater Fish, 22(2):192-201. https://doi.org/10.1111/eff.12014

Hudson, A. G., Vonlanthen, P., Müller, R., \& Seehausen, O. (2007). Review: The geography of speciation and adaptive radiation in coregonines. Advances in Limnology, 60:111-146.

Johansson, P. (2017). Temporal spawning divergence in European whitefish (Coregonus lavaretus): The first steps towards reproductive isolation in young populations (70-171 years) (Master thesis). Retrieved from http://www.divaportal.org/smash/record.jsf?pid=diva2\%3A1148367\&dswid=-4905

Jombart, T. (2008). adegenet: a R package for the multivariate analysis of genetic markers. Bioinformatics, 24(11):1403-1405. https://doi.org/10.1093/bioinformatics/btn129

Kahilainen, K. K., Lehtonen, H., \& Könönen, K. (2003). Consequence of habitat segregation to growth rate of two sparsely rakered whitefish (Coregonus lavaretus (L.)) forms in a subarctic lake. Ecology of Freshwater Fish, 12:275-285. https://doi.org/10.1046/j.1600-0633.2003.00029.x 
Kahilainen, K., Alajärvi, E., \& Lehtonen, H. (2005). Planktivory and diet-overlap of densely rakered whitefish (Coregonus lavaretus (L.)) in a subarctic lake. Ecology of Freshwater Fish, 14(1):50-58. https://doi.org/10.1111/j.1600-0633.2004.00075.x

Kahilainen K. K., \& Østbye, K. (2006). Morphological differentiation and resource polymorphism in three sympatric whitefish Coregonus lavaretus (L.) forms in a subarctic lake. Journal of Fish Biology, 68(1):63-79. https://doi.org/10.1111/j.0022-1112.2006.00876.x

Kahilainen, K. K., Patterson, W. P., Sonninen, E., Harrod, C., \& Kiljunen, M. (2014). Adaptive radiation along a thermal gradient: Preliminary results of habitat use and respiration rate divergence among whitefish morphs. PLoS ONE, 9(11): e112085.

https://doi.org/10.1371/journal.pone.0112085

2. Keller, I., \& Seehausen, O. (2012). Thermal adaptation and ecological speciation. Molecular Ecology, 21(4):782-799. https://doi.org/10.1111/j.1365-294X.2011.05397.x

Lenth, R. V. (2016a). estimability: Tools for assessing estimability of linear predictions [R package version 1.2]. https://CRAN.R-project.org/package=estimability

Lenth, R. V. (2016b). Least-Squares Means: The R Package lsmeans. Journal of Statistical Software, 69(1):1-33. doi:10.18637/jss.v069.i01

McQuinn, I. H. (1989). Identification of spring- and autumn spawning herring (Clupea harengus 
harengus) using maturity stages assigned from a gonadosomatic index model. Canadian Journal of Fisheries and Aquatic Sciences, 46(6): 969-980. https://doi.org/10.1139/f89-126

Ohlberger, J., Mehner, T., Staaks, G., \& Hölker, F. (2008) Temperature-related physiological adaptations promote ecological divergence in a sympatric species pair of temperate freshwater fish, Coregonus spp. Functional Ecology, 22(3):501-508. https://doi.org/10.1111/j.13652435.2008.01391.x

Ohlberger, J., Brännström, Å., \& Dieckman, U. (2013). Adaptive phenotypic diversification along a temperature-depth gradient. The American Naturalist, 182(3):359-373.

https://doi.org/10.1086/671169

Østbye, K., Bernatchez, L., Næsje, T. F., Himberg, K. J. M., \& Hindar, K. (2005a). Evolutionary history of the European whitefish Coregonus lavaretus (L.) species complex as inferred from mtDNA phylogeography and gill-raker numbers. Molecular Ecology, 14(14):4371-4387. https://doi.org/10.1111/j.1365-294X.2005.02737.x

Østbye, K., Næsje, T. F., Bernatchez, L., Sandlund, O. T., \& Hindar, K. (2005b). Morphological divergence and origin of sympatric populations of European whitefish (Coregonus lavaretus L.) in Lake Femund, Norway. Journal of Evolutionary Biology, 18(3):683-702.

https://doi.org/10.1111/j.1420-9101.2004.00844.x

Østbye, K., Amundsen, P.-A., Bernatchez, L., Klemetsen, A., Knudsen, R., Kristoffersen, R., Næsje, T.F., \& Hindar, K. (2006). Parallel evolution of ecomorphological traits in the European 
whitefish Coregonus lavaretus (L.) species complex during postglacial times. Molecular Ecology, 15(13):3983-4001. https://doi.org/10.1111/j.1365-294X.2006.03062.x

Præbel, K., Knudsen, R., Siwertsson, A., Karhunen, M., Kahilainen, K. K., Ovaskainen, O., Østbye, K., Peruzzi, S., Fevolden S.-E., \& Amundsen, P.-A. (2013a). Ecological speciation in postglacial European whitefish: rapid adaptive radiations into the littoral, pelagic, and lake habitats. Ecology and Evolution, 3(15):4970-4986. https://doi.org/10.1002/ece3.867

Præbel, K., Westgaard, J. I., Amundsen, P.-A., Siwertsson, A., Knudsen, R., Kahilainen, K. K., \& Fevolden, S.-E. (2013b). A diagnostic tool for efficient analysis of population structure, hybridization and conservation status of European whitefish (Coregonus lavaretus (L.)) and vendace (C. albula (L.)). Advances in Limnology, 64:247-255. https://dx.doi.org/10.1127/1612$166 X / 2013 / 0064-0026$

R Core Team (2016). R: A language and environment for statistical computing. R Foundation for Statistical Computing, Vienna, Austria. URL: https://www.R-project.org/

Redenbach, Z., \& Taylor, E. B. (2003). Evidence for bimodal hybrid zones between two species of char (Pisces: Salvelinus) in northwestern North America. Journal of Evolutionary Biology, 16(6):1135-1148.

Rice, W. R. (1989). Analyzing Tables of Statistical Tests. Evolution, 43(1):223-225. DOI: $10.2307 / 2409177$ 
Ritchie, M. G. (2007). Sexual selection and speciation. Annual Review of Ecology, Evolution and Systematics, 38:79-102. https://doi.org/10.1146/annurev.ecolsys.38.091206.095733

Rösch, R. (2000). Gonadosomatic Index (GSI) of Female Whitefish (Coregonus lavaretus) in Lake Constance. Limnologica, 30:193-196.

Rousset, F. (2007). Genepop'007: a complete reimplementation of the Genepop software for Windows and Linux. Molecular Ecology Resources, 8(1):103-106. https://doi.org/10.1111/j.14718286.2007.01931.x

Rundle, H. D., \& Nosil, P. (2005). Ecological speciation. Ecology Letters, 8(3):336-352. https://doi.org/10.1111/j.1461-0248.2004.00715.x

Schluter, D. (1996). Adaptive Radiation Along Genetic Lines of Least Resistance. Evolution, 50(5):1766-1774. https://doi.org/10.1111/j.1558-5646.1996.tb03563.x

Schluter, D. (2000). The Ecology of Adaptive radiation. Oxford University Press, U.K.

Schluter, D. (2001). Ecology and the origin of species. Trends in Ecology \& Evolution, 16(7):372380. https://doi.org/10.1016/S0169-5347(01)02198-X

Siwertsson, A., Knudsen, R., Kahilainen, K. K., Præbel, K., Primicerio, R., \& Amundsen, P.-A. (2010). Sympatric diversification as influenced by ecological opportunity and historical contingency in a young species lineage of whitefish. Evolutionary Ecology Research, 12(8):929- 
947.

Siwertsson, A., Knudsen, R., Præbel, K., Adams, C. E., Newton, J., \& Amundsen, P.-A. (2013).

Discrete foraging niches promote ecological, phenotypic, and genetic divergence in sympatric whitefish (Coregonus lavaretus). Evolutionary Ecology, 27(3):547-564.

https://doi.org/10.1007/s10682-012-9607-x

Svärdson, G. (1979). Speciation of Scandinavian Coregonus. Report of the Institute of Freshwater Research, 57:3-95.

Taylor, E. B. (1999). Species pairs of north temperate freshwater fishes: Evolution, taxonomy, and conservation. Reviews in Fish Biology and Fisheries, 9(4):299-324.

https://doi.org/10.1023/A:1008955229420

Taylor, R. S., \& Friesen, V. L. (2017). The role of allochrony in speciation. Molecular Ecology, 26(13):3330-3342. https://doi.org/10.1111/mec.14126

Valdés, P., García-Alcázar, A., Abdel, A., Arizcun, M., Suárez, C., \& Abellan, E. (2004). Seasonal Changes on Gonadosomatic Index and Maturation Stages in Common Pandora Pagellus erythrinus (L.). Aquaculture International, 12(4-5):333-343.

https://doi.org/10.1023/B:AQUI.0000042136.91952.9e 
van Oosterhout, C., Hutchinson, W. F., Wills, D. P. M., \& Shipley, P. (2004). Micro-checker: software for identifying and correcting genotyping errors in microsatellite data. Molecular Ecology Notes, 4(3):535-538. https://doi.org/10.1111/j.1471-8286.2004.00684.x

Vonlanthen, P., Roy, D., Hudson, A. G., Largiader, C.R. Bittner, D., \& Seehausen, O. (2009).

Divergence along a steep ecological gradient in lake whitefish (Coregonus sp.). Journal of Evolutionary Biology, 22(3):498-514. https://doi.org/10.1111/j.1420-9101.2008.01670.x

Wilson, J. P. F., \& Pitcher T. J. (1983). The seasonal cycle of condition in the pollan, Coregonus autumnalis pollan Thompson, of Lough Neagh, Northern Ireland. Journal of Fish Biology, 23:365370. https://doi.org/10.1111/j.1095-8649.1983.tb02916.x

\section{Authors Contribution Statement}

Conceived and designed the investigation: KP, JBT

Performed field and/or laboratory work: JBT, KH, SB, KP

Analyzed the data: JBT, KH, KP, SB

Contributed materials, reagents and/or analysis tools: JBT, KH, KP

Wrote paper: JBT, KP, KH, SB 


\section{Table 1}

Overview of the whitefish dataset including average number of gill rakers $\pm S D$; number of individuals used in FsT-calculations; number of mature individuals; average length, weight and gonad weight $\pm S D$; percentage of maturity; and average GSI for the three ecomorphs in Stuorajávri and Suohpatjávri, divided by sex. 


\begin{tabular}{|c|c|c|c|c|c|c|c|c|c|}
\hline \multirow[b]{2}{*}{ Ecomorph } & \multicolumn{9}{|l|}{ FEMALES } \\
\hline & Lake & Gill rakers $(\mathbf{N})$ & $\mathbf{N}\left(\mathbf{F s s}_{\mathbf{S T}}\right) \S$ & $\mathbf{N}(m a t u r e)$ & Length (mm) & Weight (g) & Gonad weight (g) & $\%$ Maturity & AverageGSI(g) \\
\hline \multirow[t]{2}{*}{ DR } & Stuorajávri & $34.8 \pm 2.1$ & 16 & 23 & $310 \pm 22.75$ & $236 \pm 32.48$ & $22.77 \pm 5.37$ & $85.2 \%$ & $9.59 \pm 1.34$ \\
\hline & Suohpatjávri & $39.0 \pm 2.5$ & 51 & 79 & $237 \pm 23.52$ & $89 \pm 22.89$ & $5.54 \pm 2.53$ & $97.6 \%$ & $6.35 \pm 3.05$ \\
\hline \multirow[t]{2}{*}{ LSR } & Stuorajávri & $24.4 \pm 1.6$ & 19 & 18 & $342 \pm 48.67$ & $317 \pm 152.6$ & $31.58 \pm 34.51$ & $94.7 \%$ & $8.05 \pm 8.09$ \\
\hline & Suohpatjávri & $26.5 \pm 2.4$ & 27 & 21 & $313 \pm 40.49$ & $245 \pm 117.4$ & $8.83 \pm 14.04$ & $63.6 \%$ & $3.66 \pm 4.85$ \\
\hline \multirow[t]{3}{*}{ SSR } & Stuorajávri & $22.0 \pm 1.6$ & 26 & 22 & $267 \pm 42.37$ & $154 \pm 101.8$ & $10.28 \pm 17.31$ & $75.9 \%$ & $5.14 \pm 4.38$ \\
\hline & Suohpatjávri & $23.6 \pm 3.3$ & 10 & 5 & $269 \pm 7.92$ & $134 \pm 23.93$ & $0.94 \pm 0.52$ & $50 \%$ & $0.71 \pm 0.34$ \\
\hline & \multicolumn{9}{|l|}{ MALES } \\
\hline Ecomorph & Lake & Gill rakers (N) & $\mathbf{N}(\mathbf{F s T}) \S$ & $\mathbf{N}($ mature $) \geq$ & Length (mm) & Weight (g) & Gonad weight (g) & \%Maturity & AverageGSI(g) \\
\hline \multirow[t]{2}{*}{ DR } & Stuorajávri & $34.8 \pm 2.1$ & 31 & 50 & $317 \pm 13.26$ & $241 \pm 30.76$ & $3.35 \pm 0.90$ & $100 \%$ & $1.39 \pm 0.33$ \\
\hline & Suohpatjávri & $39.0 \pm 2.5$ & 18 & 27 & $232 \pm 24.65$ & $85.5 \pm 27.21$ & $0.83 \pm 0.34$ & $100 \%$ & $0.96 \pm 0.15$ \\
\hline \multirow[t]{2}{*}{ LSR } & Stuorajávri & $24.4 \pm 1.6$ & 26 & 26 & $348 \pm 28.46$ & $331 \pm 99.81$ & $4.55 \pm 2.40$ & $93.1 \%$ & $1.32 \pm 0.29$ \\
\hline & Suohpatjávri & $26.5 \pm 2.4$ & 24 & 14 & $296 \pm 42.35$ & $207 \pm 122$ & $2.47 \pm 2.14$ & $67.9 \%$ & $1.12 \pm 0.31$ \\
\hline \multirow[t]{2}{*}{ SSR } & Stuorajávri & $22.0 \pm 1.6$ & 25 & 21 & $259 \pm 32.79$ & $131 \pm 53.30$ & $1.32 \pm 0.71$ & $85.2 \%$ & $1.00 \pm 0.22$ \\
\hline & Suohpatjávri & $23.6 \pm 3.3$ & 2 & $1 \dagger$ & $356 \dagger$ & $422 \dagger$ & $3.12 \dagger$ & $50 \% \dagger$ & $0.74 \dagger$ \\
\hline
\end{tabular}

0 Abbreviations: DR = densely rakered whitefish, LSR: large sparsely rakered whitefish, SSR = small sparsely rakered whitefish.

1 §Both mature and immature individuals were genotyped, but not all individuals. Therefore, the number of individuals can vary from the

2 number of mature individuals used for the GSI-calculations.

$3 ¥$ ¥Outlier males were removed from the dataset and is therefore not included here. A total of 139 mature males.

4 †Only one mature male SSR, therefore averages and percentages calculated for this ecomorph is not correct. We kept the values to display

5 the one male we did include in the study. 


\section{$6 \quad$ Figure 1}

7 Drawings of the three ecomorphs of European whitefish (Coregonus lavaretus L.) from northern

8 Norway and their respective gill rakers: (A) DR, densely rakered, (B) LSR, large sparsely rakered,

9 and (C) SSR, small sparsely rakered ecomorph (line drawings modified from Harrod et al. 2010).

10 The photos show three examples of female gonads for each ecomorph at the exact same time of

11 year in Suohpatjávri: DR (mature, mature, immature) LSR (mature, immature, immature) SSR

12 (mature, immature, immature). The GSI-index values calculated as a measure of sexual maturity,

13 which account for any size differences between fish, are provided next to the corresponding gonad.

\section{Table 2}

17 Fst-Table of all genotyped individuals. Below diagonal are pairwise Fst-values, above diagonal are

18 P-values.

\begin{tabular}{|c|c|c|c|c|c|c|}
\hline & StD & StL & StS & SuD & SuL & SuS \\
\hline StD & & 0.001 & 0.001 & 0.014 & 0.001 & 0.001 \\
\hline StL & 0.049 & & 0.005 & 0.001 & 0.030 & 0.001 \\
\hline StS & 0.035 & 0.016 & & 0.001 & 0.005 & 0.008 \\
\hline SuD & 0.012 & 0.059 & 0.042 & & 0.001 & 0.001 \\
\hline SuL & 0.037 & 0.011 & 0.017 & 0.047 & & 0.008 \\
\hline SuS & 0.038 & 0.020 & 0.016 & 0.032 & 0.014 & \\
\hline
\end{tabular}

19 Abbreviation of populations are: St (Stuorajávri), Su (Suohpatjávri), D (DR whitefish), L (LSR

20 whitefish), S (SSR whitefish). Code is a combination of lake and morph name. 


\section{$21 \quad$ Figure 2a and 2b:}

22 Plots showing the differences in maturity of the whitefish ecomorphs, for both sexes and across two

23 lakes. This was done using least-square means for the specified factors in the linear model and $p$ -

24 values were adjusted for multiple comparisons by Tukey's HSD. Boxes indicate the least-square

25 mean of $\log 10$ (GSI) and error bars indicate the 95\% confidence interval of the least-square mean.

26 Means sharing a letter have no statistically significant difference at the alpha level 0.05 .

27

28 


\section{Appendix}

\section{$30 \quad$ Table S1}

\section{NUMBER OF ALLELES PER POPULATION}

\begin{tabular}{|l|c|c|c|c|c|c|c|}
\hline Locus ID & Total & StD & StL & StS & SuD & SuL & SuS \\
\hline BWF2a & 5 & 3 & 3 & 3 & 4 & 3 & 3 \\
\hline BFRO-018b & 3 & 1 & 2 & 1 & 2 & 3 & 2 \\
\hline
\end{tabular}

\begin{tabular}{|lcccccccc|}
\hline ClaTet01c & 3 & 1 & 2 & 1 & 2 & 3 & 2 \\
\hline ClaTet03c & 11 & 7 & 6 & 6 & 5 & 9 & 5 \\
\hline ClaTet09c & 13 & 4 & 6 & 5 & 8 & 6 & 3 \\
\hline
\end{tabular}

\begin{tabular}{|l|ccccccc|c|}
\hline ClaTet09c & 13 & 8 & 10 & 9 & 7 & 12 & 6 \\
\hline ClaTet10c & 21 & 11 & 12 & 12 & 16 & 17 & 8 \\
\hline ClaTet05c & 11 & 5 & 7 & 6 & 3 & 9 & 3 \\
\hline
\end{tabular}

\begin{tabular}{|l|ccccccc|}
\hline ClaTet05c & 11 & 5 & 7 & 6 & 3 & 9 & 3 \\
\hline ClaTet12c & 17 & 10 & 11 & 9 & 10 & 13 & 6 \\
\hline ClaTet13c & 7 & 6 & 6 & 7 & 6 & 6 & 5 \\
\hline ClaTet15c & 5 & 4 & 4 & 5 & 4 & 4 & 5 \\
\hline
\end{tabular}

\begin{tabular}{|l|ccccccc|}
\hline ClaTet17c & 15 & 8 & 9 & 7 & 10 & 10 & 4 \\
\hline ClaTet18c & 4 & 4 & 2 & 3 & 4 & 2 & 2 \\
\hline
\end{tabular}

\begin{tabular}{|llllllll|}
\hline ClaTet18c & 4 & 4 & 2 & 3 & 4 & 2 & 2 \\
\hline Cocl-Lav4c & 2 & 2 & 1 & 1 & 1 & 2 & 1 \\
\hline Cocl-Lav6c & 4 & 3 & 3 & 2 & 2 & 2 & 2 \\
\hline Cocl-Lav18d & 2 & 2 & 2 & 2 & 2 & 2 & 1 \\
\hline Cocl_Lav49d & 7 & 2 & 4 & 5 & 6 & 3 & 2 \\
\hline Cocl-Lav52d & 13 & 8 & 9 & 9 & 9 & 8 & 7 \\
\hline C2-157e & 5 & 2 & 1 & 2 & 1 & 4 & 1 \\
\hline
\end{tabular}

31 Details of microsatellite loci used for estimating genetic differentiation among whitefish

32 ecomorphs. Total number of alleles in each locus and number of alleles in each locus per

33 population. Abbreviation of populations are: St (Stuorajávri), Su (Suohpatjávri), D (DR whitefish),

34 L (LSR whitefish), S (SSR whitefish). Code is a combination of lake and morph name. a(Patton et

35 al., 1997); b(Susnik et al., 1999); c(Winkler \& Weiss, 2008); d(Rogers et al., 2004); e(Turgeon et al., 36 1999). 